\title{
Mesoporous oxide films grown on Sn foil using various anodizing modes in alkaline electrolyte
}

\author{
Tatyana Vasilyeva ${ }^{1,2 *}$ and Nikolai Uvarov ${ }^{1,2,3}$ \\ ${ }^{1}$ Institute of Solid State Chemistry and Mechanochemistry SB RAS, Kutateladze 18, Novosibirsk, \\ 630128, Russia \\ ${ }^{2}$ Novosibirsk State University, Pirogova, 2, Novosibirsk, 630090, Russia \\ ${ }^{3}$ Novosibirsk State Technical University, Prospect Karla Marksa 20, Novosibirsk, 630073, Russia
}

\begin{abstract}
The mesoporous structures of tin oxide were obtained by a simple method of one-stage electrochemical anodizing of Sn foil in $1 \mathrm{M}$ $\mathrm{NaOH}$ in various anodizing modes. Anodizing in the pulse potentiostatic mode allowed determination of actual value of the voltage drop on the anode. For the first time the possibility of obtaining mesoporous oxide films on the surface of tin using galvanostatic mode of anodizing was demonstrated. It was found that the surface morphology of the obtained tin oxide layers is strongly dependent on the anodizing mode. Based on the data obtained, a two-stage mechanism was proposed for the growth of porous structures on the surface of tin in the galvanostatic mode including initial formation of the layer of $\mathrm{SnO} \cdot \mathrm{xH}_{2} \mathrm{O}$ which is subsequently oxidized to $\mathrm{SnO} 2 \cdot \mathrm{xH}_{2} \mathrm{O}$ mesoporous layer.
\end{abstract}

\section{Introduction}

Anodizing is a simple, perspective, environmentally friendly, cost effective and easily scalable method for producing porous oxide structures on the surface of various metals. Currently, the anodizing technique is widely applied to the production of highly ordered nanoporous, nanotubular and nanochannel oxide structures on the surface of $\mathrm{Al}$, Ti and $\mathrm{Ta}$ [1-3] as such nanostructured metal oxides have unique physical and chemical properties.

It was found that mesoporous films are also formed during the anodizing of tin [4]. Anode films based on $\mathrm{SnO}_{2}$ have great potential for use as electrode materials in solid-state gas sensors [5, 6], Li- or Na-ion batteries [7, 8], supercapacitors [9], UV detectors [10], transistors [ 11], solar cells [12], as well as carriers for catalysts in organic synthesis [13]. Therefore, the study of the formation of porous oxide layers on the surface of the tin and study of factors affecting the quality of the anodic coatings is an urgent task.

It is known that the morphology, composition and structure of anodic oxide films are strongly influenced by anodizing conditions, such as the applied potential, composition and concentration of the electrolyte, the presence of alloying additives, electrolyte stirring, temperature, purity and morphology of the original foil, as well as the geometry of the electrochemical cell. It has been proven that the use of a horizontal cell prevents mechanical deformation, change in the shape of the tin foil and destruction of the oxide 
layer [13]. At the moment it is known that it is possible to synthesize irregular mesoporous layers of tin oxide by simple one-stage potentiostatic anodizing of $\mathrm{Sn}$ in acidic [4, 14-16], alkaline [16-18] and in organic electrolytes containing $\mathrm{F}^{-}$and $\mathrm{S}^{2-}$ ions [6]. However, there have been no systematic studies of the growth of mesoporous oxide films of tin during anodizing in pulsed and galvanostatic modes.

In the present work the growth of porous layers of tin oxide on the surface of tin foil was studied in different modes of anodizing in an alkaline electrolyte. Particular attention was paid to the analysis of the morphology of the anodic films obtained under various conditions of anodizing and a mechanism for the formation of mesoporous tin structures in the galvanostatic mode was proposed.

\section{Experimental}

Layered oxide structures of tin surface were obtained by one-stage anodizing processes in an alkaline electrolyte $1 \mathrm{M} \mathrm{NaOH}$. For anodic oxidation of $\mathrm{Sn}$ foil (GOST 18394-73, thickness $0.04 \mathrm{~mm}$ ), a Teflon cell with a horizontal arrangement of electrodes was used. It is known that the purity and morphology of the original surface has a significant effect on the anodizing process, the composition of the anode layer and properties of the final products. Before anodizing, the surface of the tin was cleaned from chemical and industrial contaminants. The purification was carried out with solutions of ethanol $\mathrm{C}_{2} \mathrm{H}_{5} \mathrm{OH}$ in distilled water, then the sample was dried at room temperature.

To investigate possible influence of the counter electrode on properties of the anode layer, a three-electrode system was used in which silver chloride electrode served as the reference. In the pulsed anodizing mode, a special-form signal generator AKIP-3409/1 was taken as a voltage source. During each anodizing process, the current values were measured using a V7-78/3 voltmeter and recorded using a computer program into a file for further processing. Anodizing in galvanostatic mode was carried out using an electrochemical workstation ZIVE SP2.

The morphology of the resulting anode films was investigated by scanning electron microscopy (SEM) with a HITACHI TM 1000 electron microscope. The chemical composition of the films was determined using an Oxford Instruments TM1000 EDS detector.

\section{Results and Discussion}

\subsection{Anodizing in a pulse voltage mode}

In the present work, a series of comparative experiments was carried out with two different cathode materials, stainless steel and graphite. Measurements were done with a threeelectrode system, in which a silver chloride electrode served as a reference electrode. Studies were conducted in a pulsed mode using a pulse generator at a voltage of $4 \mathrm{~V}$, frequency of $100 \mathrm{MHz}$ and a duty cycle of $50 \%$. The shape of the signals was a meander. Voltmeter recorded voltage distribution and current response in the output file.

Fig. 1 shows a series of voltage pulses, the voltage between $\mathrm{Ag} / \mathrm{AgCl}$ electrode and the anode is shown by black and red curves for a graphite of stainless steel cathode, respectively. It can be seen that the cathode material affects the anodizing process due to different distribution of potentials on the electrodes. The voltage value at the sample changes smoothly due to the electrode polarization. Consequently, measurements in a pulsed mode using a three-electrode system make it possible to evaluate the effect of the 
cathode material on the entire anodizing process, as well as to estimate the value of the real anodizing voltage applied to the anode.

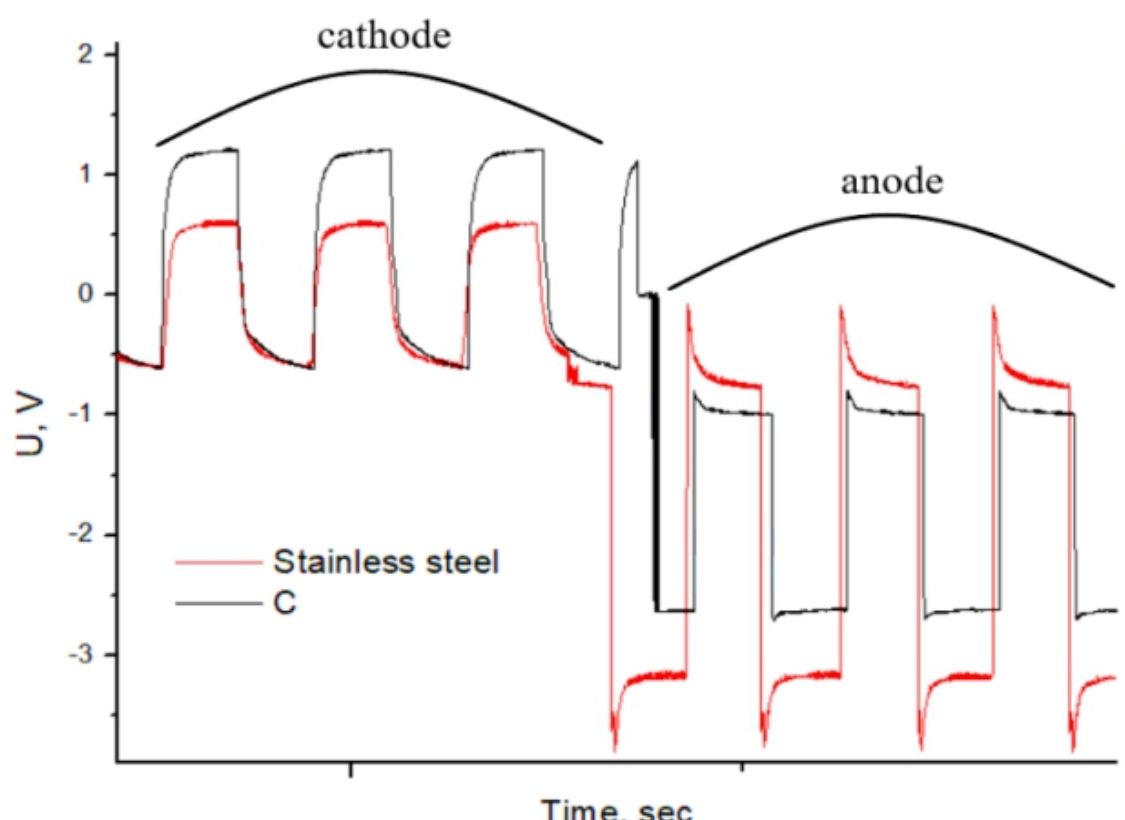

Fig. 1. Voltage drops at the cathode and anode vs. $\mathrm{Ag} / \mathrm{AgCl}$ reference electrode, the applied voltage is $4 \mathrm{~V}$.

It turned out that although the polarization is lower when a graphite is used as a cathode, the actual voltage applied to the anode is higher when the stainless steel cathode is used (Table 1).

Table 1. Distribution of the applied potential $4 \mathrm{~V}$ between the electrodes.

\begin{tabular}{|c|c|c|}
\hline Electrode & Stainless steel & Graphite \\
\hline Cathode & $1.3 \mathrm{~V}$ & $1.9 \mathrm{~V}$ \\
\hline Tin foil anode & $2.7 \mathrm{~V}$ & $2.1 \mathrm{~V}$ \\
\hline
\end{tabular}

Further experiments were carried out on anodizing in a pulsed mode with different cathode materials in an alkaline electrolyte $1 \mathrm{M} \mathrm{NaOH}$ for 10 minutes and a pulse frequency of 100 $\mathrm{mHz}$. It was also shown that the material of the counter electrode affected the morphology of the anode tin coatings. As seen from the electron microscopic images, when graphite is used as a cathode, the anodized surface of tin is rough and has a lot of macroscopic defects (Fig. 2, bottom row). When using stainless steel as the counter cathode, the surface of tin is rather smooth and pores seem to be formed on a flat area.

However, anodizing in a pulsed mode does not lead to the formation of defect-free smooth films. Perhaps this is due to the relatively low values of the voltage drop at anode, and the rate of chemical etching prevails over the electrochemical oxidation. 

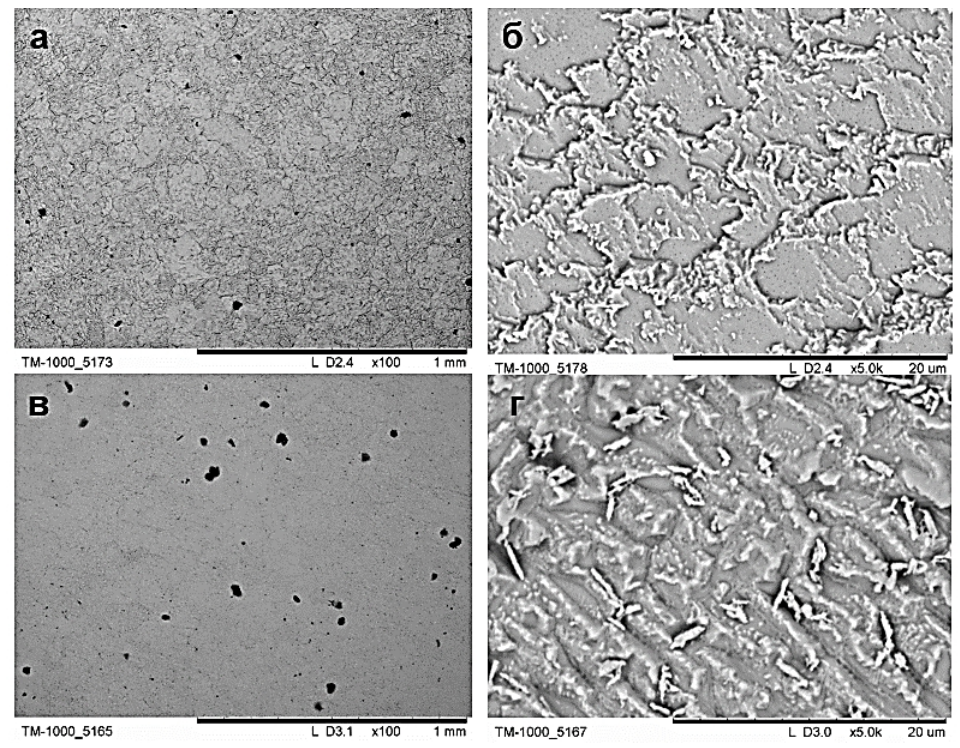

Fig. 2. Scanning electron images of tin surface after anodizing in a pulsed mode with a counter electrode made of stainless steel (top) or graphite (bottom).

\subsection{Galvanostatic mode of anodizing}

When anodizing metals such as $\mathrm{Al}$ or $\mathrm{Ti}$, the galvanostatic mode is often used as in this case the rate of growth of oxide nanostructures on a metal surface may be easily controlled. As far as we know, there are no published data on the tin anodizing in galvanostatic mode.

In this work, for the first time, anodic oxidation of tin was carried out at a constant current density values in the range from 10 to $30 \mathrm{~mA} / \mathrm{cm}^{2}$. Such current values are close to the average stationary current density values obtained during anodizing in a potentiostatic mode in $1 \mathrm{M} \mathrm{NaOH}$ electrolyte [16]. During anodizing in the galvanostatic mode the value of the current passing between the electrodes is fixed, and the voltage values are measured (Fig. 3).

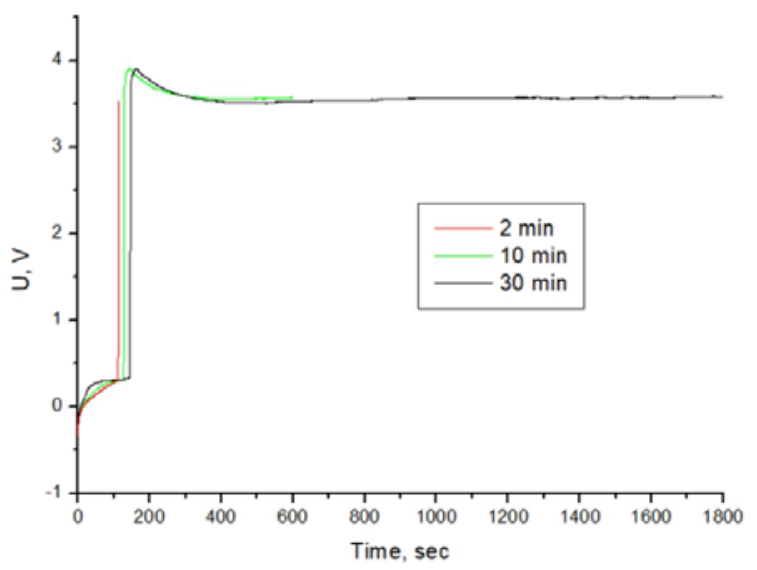

Fig. 3. The change in voltage between the electrodes during anodizing of $\mathrm{Sn}$ foil in a galvanostatic mode at a current density of $10 \mathrm{~mA} / \mathrm{cm}^{2}$. The curves correspond to 3 independent experiments on anodizing for different time. 
The curves shown in Fig. 3 and obtained at different time of anodic oxidation of the tin practically coincide, indicating a good reproducibility of the experimental data.

According to the data presented in Fig. 3, the process of anodizing proceeds through two stages. At the first stage the following electrochemical reaction takes place

$$
\mathrm{Sn}+2 \mathrm{OH}^{-}+(\mathrm{x}-1) \mathrm{H}_{2} \mathrm{O} \rightarrow \mathrm{SnO} \cdot \mathrm{xH}_{2} \mathrm{O}+2 \mathrm{e}^{-}
$$

with formation of an intermediate layer of hydrated forms of $\mathrm{SnO}$ with general formula $\mathrm{SnO} \cdot \mathrm{xH}_{2} \mathrm{O}$. This layer has a relatively low resistance, therefore the voltage value measured on this stage is also low. As the film grows, its resistance increases, leading to an increase in voltage. After a threshold voltage is reached, the $\mathrm{SnO} \cdot \mathrm{xH}_{2} \mathrm{O}$ film is oxidized with the formation of a mesoporous dielectric layer with a general formula of $\mathrm{SnO}_{2} \cdot \mathrm{xH}_{2} \mathrm{O}$ :

$$
\mathrm{SnO} \cdot \mathrm{xH}_{2} \mathrm{O}+2 \mathrm{OH}^{-} \rightarrow \mathrm{SnO}_{2} \cdot \mathrm{xH}_{2} \mathrm{O}+2 \mathrm{e}^{-}
$$

These stages are also observed during anodizing in potentiostatic mode at low voltages. After synthesis, the surface morphology of the samples was investigated using SEM, the results are shown in Fig. 4.
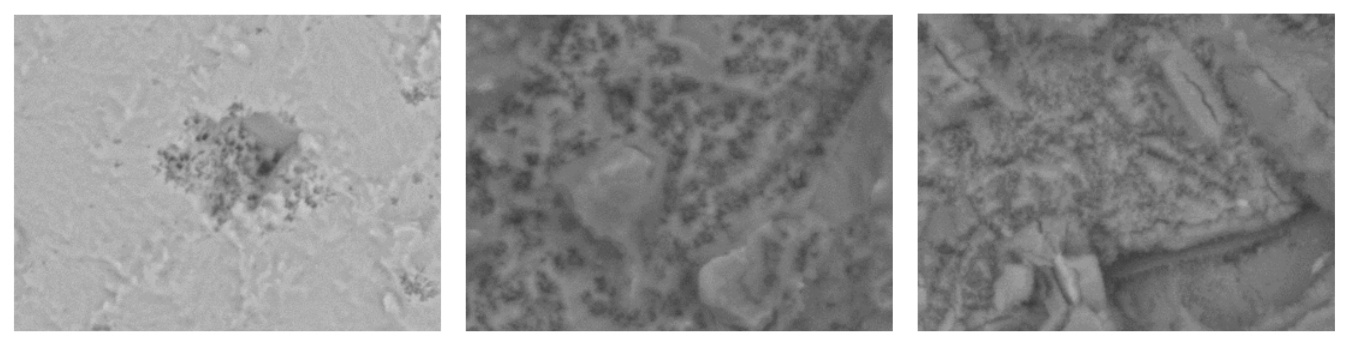

Fig. 4. Images of samples obtained in galvanostatic mode for 2, 10 and 30 minutes of anodization (scale of micrographs $10 \mu \mathrm{m}$ ).

It is seen, that at the first stage the film grows not uniformly. In the second stage the mesoporous layer is formed. However, with an increase in the duration of the anodizing process, the number of defects on the surface increases, and the pore size is so small that their size is difficult to estimate.

\section{Conclusions}

It is shown that using a three-electrode cell, one can estimate the polarization time and voltage distribution between the electrodes, determine actual voltage drop on the anode and select the optimal cathode material for anodizing. Howevr, the use of a pulsed voltage mode does not make it possible to obtain smooth mesoporous tin oxide films.

For the first time, studies of tin anodization of tin in galvanostatic mode have been carried out. Based on the results obtained by anodizing in galvanotstatic mode, the mechanism of the electrochemical oxidation with formation of an oxide layer on a tin substrate is proposed and two main stages are revealed, namely:

- during the first 150 seconds, on the surface of tin a thin barrier layer of $\mathrm{SnO} \cdot \mathrm{xH}_{2} \mathrm{O}$ is formed with relatively low resistance, as evidenced by the low potential values;

- after reaching a critical thickness of the initial $\mathrm{SnO} \cdot \mathrm{xH}_{2} \mathrm{O}$ layer, an insulating layer of $\mathrm{SnO}_{2} \cdot \mathrm{xH}_{2} \mathrm{O}$ starts to be formed that leads to an abrupt increase in the voltage up to some limiting value. The fact that the voltage reaches a stationary value indicates to a stationary 
growth of a mesoporous oxide layer of amorphous hydrated tin dioxide $\mathrm{SnO}_{2} \cdot \mathrm{xH}_{2} \mathrm{O}$ on the tin surface.

The work was supported by the state assignment of the Institute of Solid State Chemistry and Mechanochemistry Siberian Branch of the Russian Academy of Sciences, project FWUS 0237-20210007 and Russian Fund of Basic Research, grant 18-29-11054mk

\section{References}

1. H. Masuda, K. Fukuda, Science 268 (1995).

2. V. Zwilling, M. Aucouturier, E. Darque-Ceretti, Electrochim. Acta 45 (1999).

3. H.A. El-Sayed, V.I. Birss, Nano Lett. 9 (2009).

4. H.-C. Shin, J. Dong, M. Liu, Adv. Mater. 16 (2004).

5. H. R. Kim, K. I. Choi, J. H. Lee, S. A. Akbar, Sens. Actuators B Chem. 136 (2009).

6. A. Palacios-Padros, M. Altomare, A. Tighineanu, R. Kirchgeorg, N. K. Shrestha, I. Diez-Perez, F. Caballero-Briones, F. Sanz, P. Schmuki, J. Mater. Chem. A 2 (2014)

7. Y. Han, X. Wu, Y. Ma, L. Gong, F. Qu, H. Fan, CrystEngComm. 13 (2011).

8. H. Bian, J. Zhang, M.-F Yuen, W. Kang, Y. Zhan, D. Y. W Yu, Zh. Xu, Y. Y. Li, J. Power Sources 307 (2016).

9. D. V. Shinde, D. Y. Lee, S. A. Patil, I. Lim, S. S. Bhande, W. Lee, M. M. Sung, R. S. Mane, N. K. Shrestha, S.-H. Han, RSC Adv. 3 (2013).

10. L. Chen, X. Li, Y. Wang, C. Gao, H. Zhang, B. Zhao, F. Teng, J. Zhou, Z. Zhang, X. Pan, E. Xie, J. Power Sources 272 (2014).

11. G. Shin, Chang H. Yoon, M. Y. Bae, Y. Ch. Kim, S. K. Hong, J. A. Rogers, J. S. Ha, Small 7 (2011).

12. Granqvist C.G., Sol. Energy Mater Sol. Cells 91 (2007).

13. H.-S. Oh, H. N. Nong, P. Strasser, Adv. Funct. Mater. 25 (2015).

14. L. Zaraska, M. Bobruk, G.D. Sulka, Adv. Condens. Matter Phys. 1-11 (2015).

15. L. Zaraska, K. Gawlak, M. Gurgul, D. Gilec, M. Koziel, R. P. Socha, G.D. Sulka, Surf. Coat. Technol. 362 (2019).

16. T.A. Vasilieva, N.F. Uvarov, B.B. Bokhonov, Mater. Today Proc. 25, 401 (2020).

17. S. Ono, Y. Kobayashi, R. Kobayashi, H. Asoh, ECS Trans. 16 (2008).

18. J. J. Teh, G. H. Guai, X. Wang, K. Ch. Leong, Ch. M. Li, P. Chen, Renew. Sust. Energ. Rev. 5 (2013). 